A Sadeghi-Tari ${ }^{1}$, M Jamshidian-Tehrani ${ }^{1}$, A Nabavi ${ }^{1}$, S Sharif-Kashani ${ }^{2}$, E Elami ${ }^{1}$, N Hassanpour ${ }^{1}$ and

\section{KA Zamani ${ }^{1}$}

${ }^{1}$ Eye Research Center, Farabi Eye Hospital, Tehran University of Medical Sciences, Tehran, Iran

${ }^{2}$ Department of Radiology, Tehran University of

Medical Sciences, Imam Khomeini Hospital, Tehran,

Iran

E-mail: mansooreh_2468@yahoo.com

Eye (2017) 31, 814-815; doi:10.1038/eye.2016.291; published online 23 December 2016

\section{Sir,}

Identification of a novel NRL mutation in a Chinese

family with retinitis pigmentosa by whole-exome sequencing

Retinitis pigmentosa (RP) is a group of inherited retinal degenerative disorders affecting $\sim 1$ in 4000 individuals worldwide. ${ }^{1}$ Because of the great genetic heterogeneity of $\mathrm{RP}$, technologies based on the next-generation sequencing are increasingly used to screen mutations or identify novel causative genes in RP patients for investigative and diagnostic purposes. ${ }^{2}$ Here, we report a Chinese family suffering from autosomal dominant RP (adRP). The genetic cause of the family was further investigated.

The proband (II:2 in Figure 1a, 43 years old) complained of night blindness since childhood, followed by visual field loss and reduction of visual acuity. Fundus examination revealed bone-spicules pigmentation in the mid-peripheral retina (Figure 1b). Her 14-year-old daughter (III:1) also reported night blindness before age 10 years. No apparent pigmentation could be seen at the time of the last ophthalmologic examination (Figure 1b). To identify the possible causing mutation(s), we performed whole-exome sequencing using the proband's genomic DNA. Through a multistep bioinformatics pipeline (Supplementary Information), candidate variants were selected and validated by Sanger sequencing and segregation analysis. A novel c.147_149del (p.Ser50del) variation in NRL was identified as the most likely cause of the family (Figure 1c). The variation was absent in 250 normal controls.

The neural retina leucine zipper $(N R L)$ gene encodes a 237-aa basic motif-leucine zipper transcription factor of a

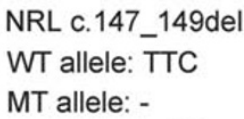

NRL c.147_149del

WT allele: TTC

MT allele: -

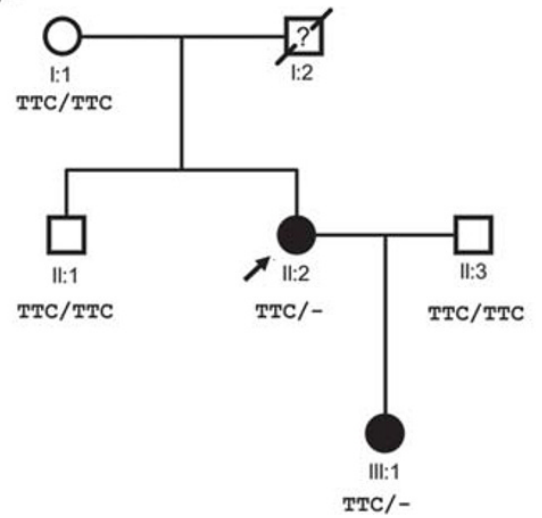

C

II:1

Normal
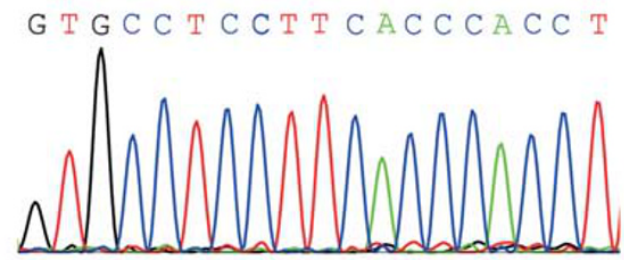

II:2

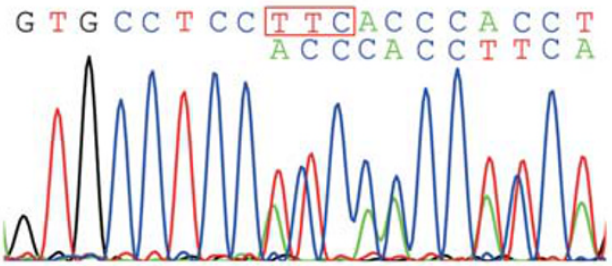

Affected

b
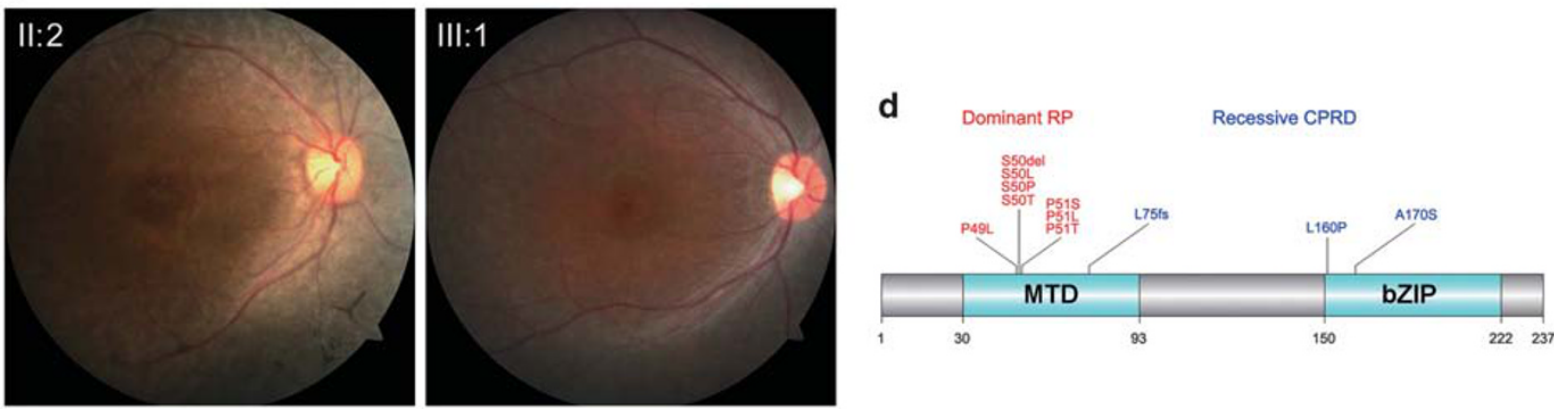

Figure 1 Identification of a novel NRL mutation in a Chinese adRP family. (a) Pedigree of the family. Circles, females; squares, males; filled symbols, affected individuals; empty symbols, unaffected individuals; arrow, proband; question mark, unexamined individual. (b) Fundus photographs of the two patients. II:2, the proband, 43 years old; III:1, 14 years old. (c) Sanger sequencing of the c.147_149delTTC mutation. The deleted nucleotides were marked with a red box. (d) Schematic structure of the NRL protein. The red color- and blue color-labeled NRL mutations associated with dominant retinitis pigmentosa and recessive clumped pigmentary retinal degeneration, respectively. 
a
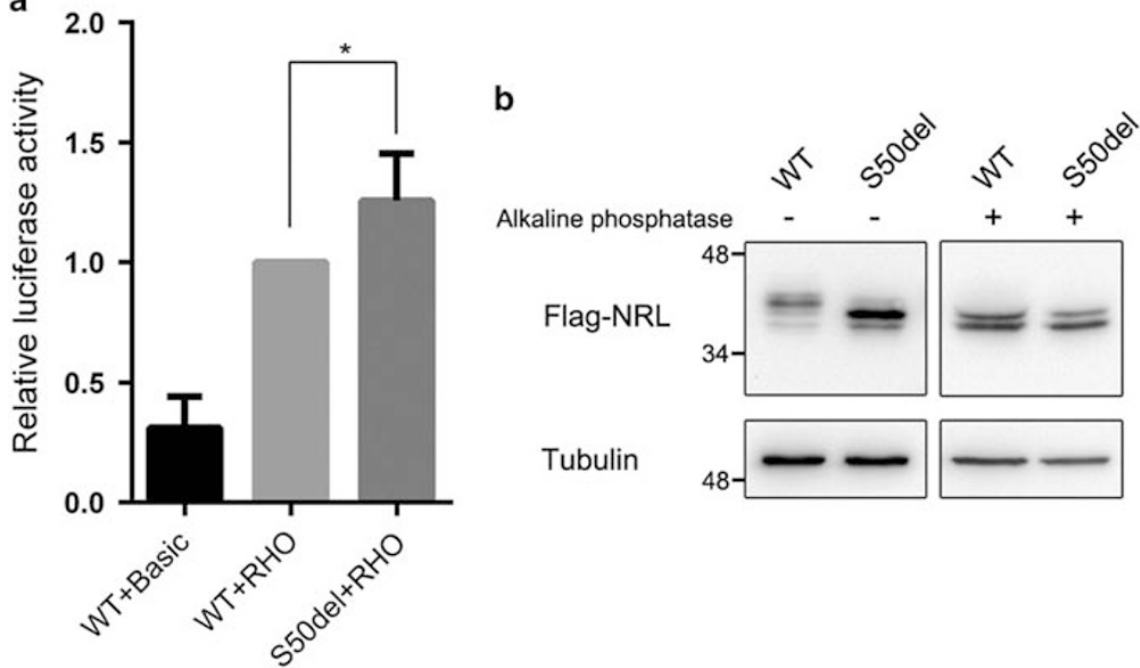

Figure 2 Effects of the S50del mutation on the transactivation ability and phosphorylation modification of NRL protein. (a) Dual-luciferase reporter assay of the WT and S50del mutant NRL proteins. Relative luciferase activities of the three groups, WT+basic, WT+RHO, and S50del $+\mathrm{RHO}$, were shown as mean \pm SD. ${ }^{*} P<0.05$. (b) Electrophoretic mobility shift assay of the Flag-tagged WT and S50del mutant NRL. Tubulin was used as a loading control. Samples treated with alkaline phosphatase were used as dephosphorylated controls.

the Maf subfamily, which has a critical role in the regulation of photoreceptor development and function. Currently, all known adRP-causing mutations in NRL are located in the Pro49, Ser50, and Pro51 codons (Figure 1d), including P49L, ${ }^{3}$ S50T, S50P, S50L, P51T, P51L, and P51S. ${ }^{4}$ These mutations in NRL have been proven to reduce the phosphorylation of NRL and increase its transactivation ability. ${ }^{4}$

To see whether the S50del mutation showed similar effects like other NRL mutations causing adRP, we first measured the transactivation abilities of WT and S50del mutant NRL on the human RHO (encoding rhodopsin) promoter ${ }^{5}$ using the dual-luciferase reporter assay. As expected, the transcriptional level of $\mathrm{RHO}$ promoter was higher when co-transfected with Ser50del mutant NRL as compared with WT NRL (Figure 2a). Furthermore, we examined the phosphorylated isoforms of WT and S50del mutant NRL protein by western blot. In the WT lane, multiple bands could be observed, which represented the phosphorylated isoforms of NRL according to previous studies. However, the S50del mutant NRL protein showed a smaller and concentrated band, indicating a remarkable reduction of phosphorylation modification (Figure $2 b$ ). After treated with alkaline phosphatase, which removes phosphate groups from proteins, both of the WT and S50del groups did not exhibit a multiple-band pattern. These results were in agree with previous studies, and further proved that the S50del mutation accelerates the transcriptional activation ability of NRL and decreases its phosphorylation level just like those known adRP-causing mutations in NRL.

In summary, through whole-exome sequencing, we identified a novel NRL mutation (c.147_149del, p.Ser50del) leading to adRP in a Chinese family, which alters the phosphorylation modification and transactivation ability of NRL. Our study widens the mutation spectrum of NRL and provides insight on the functional studies of NRL.

\section{Conflict of interest}

The authors declare no conflict of interest.

\section{Acknowledgements}

We are grateful to the family members. This study was supported by grants from the National Natural Science Foundation of China (nos 81270983, 81371064, 31471199, 31571303, and 81500762) and China Postdoctoral Science Foundation (2015M582216).

\section{References}

1 Wright AF, Chakarova CF, Abd El-Aziz MM, Bhattacharya SS. Photoreceptor degeneration: genetic and mechanistic dissection of a complex trait. Nat Rev Genet 2010; 11(4): 273-284.

2 Chiang JP, Lamey T, McLaren T, Thompson JA, Montgomery H, De Roach J. Progress and prospects of next-generation sequencing testing for inherited retinal dystrophy. Expert Rev Mol Diagn 2015; 15(10): 1269-1275.

3 Gao M, Zhang S, Liu C, Qin Y, Archacki S, Jin L et al. Whole exome sequencing identifies a novel NRL mutation in a Chinese family with autosomal dominant retinitis pigmentosa. Mol Vis 2016; 22: 234-242.

4 Kanda A, Friedman JS, Nishiguchi KM, Swaroop A. Retinopathy mutations in the bZIP protein NRL alter phosphorylation and transcriptional activity. Hum Mutat 2007; 28(6): 589-598.

5 Rehemtulla A, Warwar R, Kumar R, Ji X, Zack DJ, Swaroop A. The basic motif-leucine zipper transcription factor $\mathrm{Nrl}$ can 
positively regulate rhodopsin gene expression. Proc Natl Acad Sci USA 1996; 93(1): 191-195.

Y Qin ${ }^{1,4}, \mathrm{~F} \mathrm{Liu}{ }^{1,4}, \mathrm{~S} Y u^{1}$, L Yang ${ }^{1}, \mathrm{M}$ Gao ${ }^{1}, \mathrm{Z}$ Tang ${ }^{1}$, AY Guo ${ }^{2}, \mathrm{M}$ Zhang ${ }^{3}, \mathrm{P} \mathrm{Li}^{3}$ and $\mathrm{M} \mathrm{Liu}^{1}$

${ }^{1}$ Department of Genetics and Developmental Biology, Key Laboratory of Molecular Biophysics of Ministry of Education, College of Life Science and Technology, Huazhong University of Science and Technology, Wuhan, PR, China

${ }^{2}$ Department of Bioinformatics and Systems Biology, Key Laboratory of Molecular Biophysics of Ministry of
Education, College of Life Science and Technology, Huazhong University of Science and Technology, Wuhan, PR, China

${ }^{3}$ Department of Ophthalmology, Union Hospital, Tongji Medical College, Huazhong University of Science and Technology, Wuhan, PR, China Email: 44592236@qq.com

Presentation: Part of this work was presented at the World Ophthalmology Congress 2010, Berlin, Germany. ${ }^{4}$ These authors contributed equally to this work.

Eye (2017) 31, 815-817; doi:10.1038/eye.2016.327; published online 20 January 2017

Supplementary Information accompanies this paper on Eye website (http://www.nature.com/eye) 